\title{
Doubling of gyrotron radiation frequency due to nonlinear susceptibility in A3B5 semiconductors
}

\author{
Vladimir V. Rumyantsev ${ }^{1}$, A. A. Dubinov ${ }^{1}$, A.P. Fokin ${ }^{2}$, V.V. Utochkin ${ }^{1}$, M.Yu. Glyavin ${ }^{2}$, \\ S. V. Morozov ${ }^{1}$ \\ ${ }^{1}$ Institute for Physics of Microstructures of RAS, Nizhny Novgorod, Russian Federation, rumyantsev@ipmras.ru \\ ${ }^{2}$ Institute of Applied Physics of RAS, Nizhny Novgorod, Russian Federation
}

At present, there is much activity aimed for development and application of different sources of radiation with terahertz $(\mathrm{THz})$ frequency range, which are necessary for a variety of scientific and practical applications such as spectroscopy, radioastronomy, environmental monitoring, security and counterterrorism [1]. Powerful sources of $\mathrm{THz}$ radiation like synchrotrons and free electron lasers (FELs) have high cost and large size, which limits their use even for scientific applications. Complicated solutions are required to increase the frequency of the vacuum sources such as a backward wave oscillator up to 1 $\mathrm{THz}$ and higher [2]. Semiconductor quantum cascade lasers in $\mathrm{THz}$ range operate only at cryogenic temperatures [3-5]. Molecular lasers, although operating at room temperature, are available only for a fixed set of frequencies [6]. THz radiation sources based on femtosecond lasers exhibit an extremely broad spectrum ( $\sim 1 \mathrm{THz}$ wide), which is not always acceptable for spectroscopy applications and have rather low output power [7]. Currently, the media of choice for optical rectification are $\mathrm{LiNbO} 3$ [8], $\mathrm{ZnTe}$ [9], GaP [10] crystals. They all have fairly high losses in the THz range and the conversion efficiency even for state-of-the-art experiments is approaching $10^{-3}$ [8] and in typical cases is an order of magnitude lower.

Alternative way to obtain intense $\mathrm{THz}$ radiation is to "multiply" the frequency of incident radiation using nonlinear susceptibility in semiconductors. This approach requires intense sources at the fundamental frequency in sub-THz range, among which there were mainly molecular lasers. However, recently a sufficient progress has been made in the development of sub-THz range gyrotrons. Continuous wave (cw) gyrotrons with frequencies of $460 \mathrm{GHz}$ [11] and 527 $\mathrm{GHz}$ [12] have been demonstrated. IAP RAS recently developed unique pulse solenoids with magnetic fields up to $50 \mathrm{~T}$ that allowed implementing gyrotrons on the first cyclotron harmonic with the output frequency of up to $1.3 \mathrm{THz}$ with pulse duration $\sim 50 \mu \mathrm{s}$ [13]. On the basis of "dry" cryomagnet with magnetic fields up to $10 \mathrm{~T} \mathrm{cw}$ gyrotrons with frequencies 263 $\mathrm{GHz}$ [14] and $250 \mathrm{GHz}$ were fabricated. A generator with $889 \mathrm{GHz}$ frequency (in a synchronism with the third harmonic of the cyclotron frequency) was developed, as well as a pulsed generator with a frequency of $\sim 1 \mathrm{THz}$ and power of $\sim 10 \mathrm{~W}$ at the second harmonic of gyrofrequency [15]. These results were obtained by using the unique cryomagnet with the magnetic field of up to $20 \mathrm{~T}$. In 2017, a double-beam gyrotron at the second harmonic with frequency of $0.8 \mathrm{THz}$ was demonstrated [16].
For such sources, frequency doubling is enough to obtain frequencies above $1 \mathrm{THz}$, while previous works focused on generating the third harmonic of gyrotron radiation in $\mathrm{n}$-type $\mathrm{Ge}$ and $\mathrm{Si}$ at much lower frequencies [17, 18]. Third harmonic generation was demonstrated in Ref. [17] with the effectiveness of $0.05 \%$ under gyrotron pumping with $70 \mathrm{GHz}$ frequency, and in Ref. [18] under gyrotron pumping with 118 $\mathrm{GHz}$ frequency and $0.07 \%$ conversion efficiency. The frequency tripling is possible in semiconductor materials due to the third order nonlinearity, which is predominantly electronic (third order nonlinearity due to ionic motions is usually weaker) $[19,20]$. The frequency doubling is possible in crystals lacking the inversion centre. In this work we consider the prospects of several A3B5 semiconductors for frequency doubling due to the second order susceptibility of the crystal lattice. It should be noted that nonlinear optical properties of A3B5 materials have been quite poorly studied in far infrared region where the frequency dispersion of second order nonlinear coefficient is substantial [21 - 24]. Second-order susceptibility and corresponding frequency doubling were investigated mainly in GaAs, using $\mathrm{CH}_{3} \mathrm{~F}$ laser [21] and free electron laser [22] as the radiation sources in the spectral ranges of $0.6-1.7 \mathrm{THz}$ and $4--6 \mathrm{THz}$, respectively. However, second order nonlinearity in GaAs is rather weak as can be seen from table:

Table. Second-order susceptibility of several A3B5 semiconductors

\begin{tabular}{|l|l|}
\hline Semiconductor & Second-order susceptibility, $\chi[21,25]$ \\
\hline $\mathrm{GaAs}$ & $57 \cdot 10^{-10} \mathrm{~cm} / \mathrm{V}$ \\
\hline $\mathrm{GaP}$ & $18 \cdot 10^{-10} \mathrm{~cm} / \mathrm{V}$ \\
\hline $\mathrm{GaSb}$ & $150 \cdot 10^{-10} \mathrm{~cm} / \mathrm{V}$ \\
\hline $\mathrm{InP}$ & $345 \cdot 10^{-10} \mathrm{~cm} / \mathrm{V}$ \\
\hline $\mathrm{InAs}$ & $155 \cdot 10^{-10} \mathrm{~cm} / \mathrm{V}$ \\
\hline $\mathrm{CdTe}$ & $100 \cdot 10^{-10} \mathrm{~cm} / \mathrm{V}[26]$ \\
\hline
\end{tabular}

The intensity of the second harmonic $I_{2 v}$ can be calculated [27] as (c -- light velocity, n(v) -- refractive index at the frequency $v, \Delta n=n(v)-n(2 v))$

$$
I_{2 v}=\frac{128 \pi^{5} \chi^{2} v^{2} L^{2}}{n(v)^{2} n(2 v) c^{3}} I_{v}^{2}\left(\frac{\sin 2 \pi v \Delta n L / c}{2 \pi v \Delta n L / c}\right)^{2}
$$

Thus, the output intensity is proportional to the square of intensity at the fundamental frequency $I_{v}$ susceptibility $\chi$, fundamental frequency $v$ and sample length L. As can been seen from the Table, the susceptibility in InP is 6 times higher than in GaAs, al- 
lowing sufficient enhancement of the second harmonic intensity. Note that maximum crystal length can be limited by refractive index dispersion and the optical losses. Typical frequencies of the lattice vibrations of optical transitions related to impurity absorption are considerably higher than $1 \mathrm{THz}$ in semiconductors under consideration [25]. Therefore, the losses are mainly due to free carriers, density of which can be made negligibly low in state-of-the-art InP, reducing the absorption coefficient in the $200--800 \mathrm{GHz}$ region down to less than $1 \mathrm{~cm}^{-1}$ [28]. The refractive index change with frequency in the same range is extremely small $\Delta \mathrm{n} \sim 0.002$ [28]. Thus, the crystal of several $\mathrm{cm}$ length can be used for frequency doubling. Fig. 1 gives the comparison of estimated output power of the second harmonic for GaAs and $\operatorname{InP}$ with $\mathrm{L}=$ $5 \mathrm{~cm}$ and fundamental radiation power of $50 \mathrm{~kW} / \mathrm{cm}^{2}$.

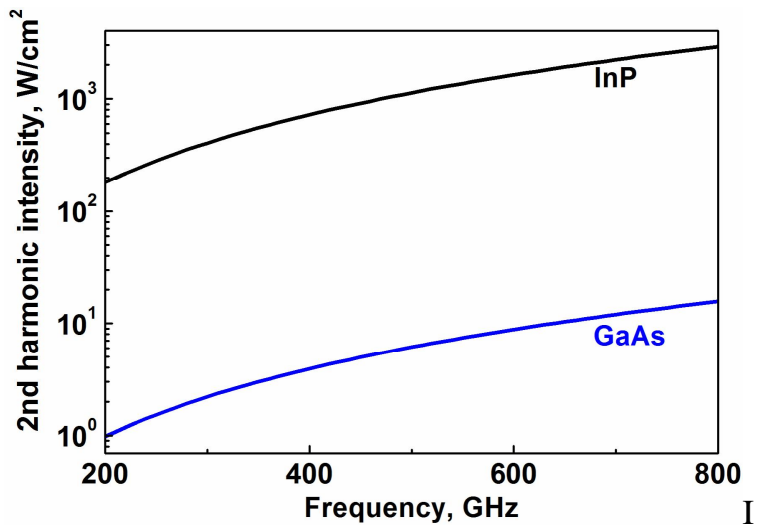

Fig. 1. The estimated second harmonic intensity vs. fundamental frequency for $5 \mathrm{~cm}$ long GaAs and InP crystals under fundamental radiation power of $50 \mathrm{~kW} / \mathrm{cm}^{2}$. Data on the refractive index for InP and GaAs is taken from Ref [28] and Ref [25], respectively

As can be seen, InP crystal is expected to provide about two-order enhancement over GaAs, studied in previous works [21-23]. We conclude that employing high-resistivity InP crystals is a promising route to obtain $\mathrm{THz}$ radiation by doubling the frequency of intense gyrotron radiation. The work was supported by Russian Science Foundation grant \#18-79-10112.

\section{References}

1. Van der Weide D. Applications and Outlook for Electronic Terahertz Technology // Optics \& Photonics News. 2003. V.14. No.4. P. 48-53

2. Xiong Xu, Yanyu Wei, Fei Shen, Hairong Yin, Jin Xu et al A watt-class 1-THz backward-wave oscillator based on sine waveguide // Phys. Plasmas. 2012. 19, 013113.

3. Williams B.S. Terahertz quantum-cascade lasers // Nature Photonics. 2007. V.1. P. 517.

4. Fathololoumi S., Dupont E., Chan C.W.I., Wasilewski Z.R., Laframboise S.R., Ban D., Matyas A., Jirauschek C., Hu Q., Liu H.C. Terahertz quantum cascade lasers operating up to $\sim 200 \mathrm{~K}$ with optimized oscillator strength and improved injection tunneling // Optics Express. 2012. 20 (4). 3866.

5. Wang X., Shen C., Jiang T., Zhan Zh., Deng $O ., L i$ W., Wu W., Yang N., Chu W., Duan S.. High-power terahertz quantum cascade lasers with $0.23 \mathrm{~W}$ in continuous wave mode. // AIP Advances. 2016. 6. 075210 .
6. Dodel G. On the history of far-infrared (FIR) gas lasers: Thirty-five years of research and application // Infrared Phys. Technol. 1999. 40 127-39

7. Lewis R.A. A review of terahertz sources // J. Phys. D: Appl. Phys. 2014. 47374001

8. Sergey B. Bodrov, Aleksey A. Murzanev, Yury A. Sergeev, Yury A. Malkov, and Andrey N. Stepanov Terahertz generation by tilted-front laser pulses in weakly and strongly nonlinear regimes // Applied Physics Letters. 2013. 103. 251103

9. Vidal S., Degert J., Tondusson M., Freysz E., and Oberlé J., Optimized terahertz generation via optical rectification in ZnTe crystals // J. Opt. Soc. Am. B. 2014. 31, 149-153.

10. Taniuchi T., Nakanishi H. Collinear phase-matched terahertz-wave generation in GaP crystal using a dual-wavelength optical parametric oscillator // Journ. Appl. Phys. 2004. v. 95, 7588.

11. Torrezan A.C., Han S.T., Mastovsky I., Shapiro M.A., Sirigiri J.R., Temkin R.J., Griffin R.G., Barnes A.B. ContinuousWave Operation of a Frequency-Tunable 460-GHz SecondHarmonic Gyrotron for Enhanced Nuclear Magnetic Resonance. // IEEETrans. 2010. V.PS-38. №6.P.1150

12. Jawla S., Nanni E., Shapiro M. et al.// 2011 36th Int. Conf. on Infrared, Millimeter and Terahertz Waves (IRMMW-THz). Houston. 2-7 Oct. 2011. N.Y.IEEE, 2011. P. DOI 10.1109. IRMMWTHz.2011.61 05096

13. Glyavin M.Yu., Ginzburg N.S., Goldenberg A.L., Denisov G.G., Luchinin A.G., Manuilov V.N., Zapevalov V.E., Zotova I.V. THz Gyrotrons: Status and Possible Optimizations // Terahertz Science and Technology, V.5, No.2, 2012

14. Glyavin M. Yu., Chirkov A.V., Denisov G.G., et al. Experimental tests of a $263 \mathrm{GHz}$ gyrotron for spectroscopic applications and diagnostics of various media. // Review of Scientific Instruments. 2015. 86. 054705.

15. Idehara T., Saito T., Ogawa I. The potential of the gyrotrons for development of the sub-terahertz and the terahertz frequency range - A review of novel and prospective applications, Proceedings of 2nd International Symposium on the Manipulation of Advanced Smart Materials // ThinSolidFilms. 2008. V. 517. No 4. P.1503, 2008

16. Idehara T., Glyavin M., Kuleshov A., Sabchevski S., Manuilov V., Zaslavsky V., Zotova I., Sedov A. A novel THz-band double-beam gyrotron for high-field DNP-NMR spectroscopy. // Rev.of Sci.Instrum. 2017. 88. 094708

17 Keilmann F., Brazis R., Barkley H., Kasparek W., Thumm M. and Erckmann V. Millimeter-Wave Frequency Tripling in Bulk Semiconductors // EPL (Europhysics Letters), Volume 11, Number 4, p. 337

18 Narkowicz R., Siegrist M.R., Moreau Ph., Hogge J.P., Raguotis R. and Brazis R. Third-Order Susceptibility of Silicon Crystals Measured with Millimeter-Wave Gyrotron // Acta Physica Polonica A. 2011. V. 119, No. 4, p. 509

19. Seeger $K$., Microwave frequency multiplication by hot electrons, // J. Appl. Phys. 1963. 34, 1608-1610.

20. Nimtz G. and Seeger K., Microwave mixing by hot electrons in homogeneous semiconductors // J. Appl. Phys. 1968. 39, 2263-2266.

21. Mayer A. and Keilmann F., Far-infrared nonlinear optics. I. $\chi^{\wedge} 2$ near ionicresonance, // Phys. Rev. B. 1986. 33, 6954-6961.

22. Dekorsy T., Yakovlev V.A., Seidel W., Helm M., and Keimann F., Infrared phonon-polariton resonance of the nonlinear susceptibility in GaAs // Phys. Rev. Lett. 2003. 90, 055508-1/4.

23. McFee J.H., Boyd G.D., Schmidt P.H. Redetermination of the nonlinear optical coefficients of Te and GaAs by comparison with Ag3SbS3 // Appl. Phys. Lett. 1970. V.17 P.57.

24. Flytzanis C. Infrared Dispersion of Second-Order Electric Susceptibilities in Semiconducting Compounds // Phys.Rev. B. 1972. v.6, 1264.

25. Madelung, Semiconductors: Data Handbook (SpringerVerlag, New York, 2003).

26. Akitt D., Johnson C., Coleman P. Nonlinear susceptibility of CdTe // IEEE Journal of quantum electronics. 1970. V. QE - 6, 496.

27. Frits Zernike \& John E. Midwinter Applied Nonlinear Optics John Wiley \& Sons Inc. 1973

28. Alyabyeva L.N., Zhukova E.S., Belkin M.A., and Gorshunov B.P. Dielectric properties of semi-insulating $\mathrm{Fe}$-doped InP in the terahertz spectral region // Scientific Reports. 2017. 7. 7360. 